\title{
Patient-Specific Surgical Implant Using Cavity-Filled Approach for Precise and Functional Mandible Reconstruction
}

\author{
Khaja Moiduddin 1,*(D), Syed Hammad Mian ${ }^{1}\left(\mathbb{D}\right.$, Wadea Ameen ${ }^{1}$, Mohammed Alkindi ${ }^{2} \mathbb{D}$, \\ Sundar Ramalingam ${ }^{2}$ and Osama Alghamdi ${ }^{2}$ \\ 1 Advanced Manufacturing Institute, King Saud University, Riyadh 11421, Saudi Arabia; \\ smien@ksu.edu.sa (S.H.M.); wqaid@ksu.edu.sa (W.A.) \\ 2 Department of Oral and Maxillofacial Surgery, College of Dentistry, King Saud University, \\ Riyadh 11545, Saudi Arabia; malkindi@ksu.edu.sa (M.A.); smunusamy@ksu.edu.sa (S.R.); \\ oghamdi@ksu.edu.sa (O.A.) \\ * Correspondence: khussain1@ksu.edu.sa; Tel.: +966-11-469-7372
}

Received: 1 July 2020; Accepted: 27 August 2020; Published: 31 August 2020

\begin{abstract}
Mandibular reconstruction is a complicated task because of the complex nature of the regional anatomy. Computer-assisted tools are a promising means of improving the precision and safety of such complex surgeries. The digital techniques utilized in the reconstruction of mandibular defects based on medical data, computer-aided-design approaches, and three-dimensional (3D) printing are widely used to improve the patient's aesthetic appearance and function, as well as the accuracy and quality of diagnosis, and surgical outcomes. Nevertheless, to ensure an acceptable aesthetical appearance and functional outcomes, the design must be based on proper anatomical reconstruction, mostly done in a virtual environment by skilled design engineers. Mirroring is one of the widely used techniques in the surgical navigation and reconstruction of mandibular defects. However, there are some discrepancies and mismatches in the mirrored anatomical models. Hence, in order to overcome these limitations in the mirroring technique, a novel approach called the cavity-filled technique was introduced. The objective of this study was to compare the accuracy of the newly recommended cavity-filled technique with the widely used mirror reconstruction technique in restoring mandibular defects. A prominent 3D comparison technique was employed in this work, where the resected and the reconstructed mandibles were superimposed to quantify the accuracy of the two techniques. From the analysis, it can be inferred that the cavity-filled technique with a root-mean-square value of $1.1019 \mathrm{~mm}$ produced better accuracy in contrast to the mirroring approach, which resulted in an error of $1.2683 \mathrm{~mm}$. Consequently, by using the proposed cavity-filled design, the discrepancy between the reconstruction plate and the bone contour was mitigated. This method, owing to its high precision, can decrease the number of adjustments and the time of surgery, as well as ensure a quick recovery time with better implant tissue in-growth.
\end{abstract}

Keywords: 3D printing; custom-specific implant; mirror reconstruction; 3D comparison; cavity-filled approach

\section{Introduction}

Functional and aesthetic defects in the maxillofacial region occur primarily due to various ailments, such as tumor excision, trauma, malformation, jaw atrophy, and benign or malignant neoplasm [1,2]. Immediate reconstruction is the most effective way to achieve improved and long-term functional outcomes [3]. Recent advances in technology and innovations in the patient-specific implants and their treatment led to the minimization of surgical time and cost. Precise design and production of 
patient-specific implants are essential in the surgical outcome. One of the biggest challenges, even for experienced surgeons, is to precisely fit the implant onto the bone contours and eliminate postoperative revisions. The success rate of any reconstructive implant surgery depends on several factors, such as medical evaluation, implant design, and the surgeon's skill [4]. The majority of conventionally manufactured reconstructive implants are of standard size and form. Such implants must be adjusted by manual bending or twisting according to the bone contour of the patient, resulting in poor adaptation and prolonged operating time [5]. Most patients undergo secondary surgical reconstruction to refine the cosmetic and functional outcomes that result in psychological stress and social implications $[6,7]$. The pre-operative preparation and three-dimensional (3D) printed patient-specific implant assessment are crucial to mitigate these instances. In addition, accurate patient-specific implants and their evaluation will result in reduced operating time, predictability of surgical outcome, and increased implant accuracy $[8,9]$. Virtual pre-operative planning and 3D-printed polymer models help minimize revision, as well as errors, eventually providing better aesthetic and functional results [10].

The technique of 3D printing developed in the mid-1980s laid the foundation for the efficient manufacturing of complex anatomical models, whereby anatomical defects can be measured and pre-planned by surgeons, and surgeries can be simulated [11]. The anatomical curvature of the skull and its facial bone is extremely complex, and it is often a demanding process for skilled surgeons to accurately align and rebuild the implant [12]. In the past, several computer-assisted design (CAD) and 3D planning strategies were proposed for the reconstruction of mandibular and dental implants $[13,14]$. These concepts utilized a preoperative virtual simulation of the proposed mandibular defect, and they could easily modify the 3D file multiple times, rather than relying heavily on the traditional intraoperative manual assessment [15]. In addition, 3D reconstruction planning improved the surgical precision, reduced the operating time, and ultimately enhanced the structural and functional outcomes [16]. Bartier et al. [17] demonstrated that a 3D reconstruction technique maintained the symmetrical accuracy, as well as improved the functional and aesthetic outcomes, in mandibular reconstruction when compared to traditional freehand technique. Mascha et al. [18] and Yang et al. [19] also developed a patient-specific mandibular reconstruction plate using a CAD procedure and proved that it was more effective and satisfying for accurate mandibular reconstruction. According to Davies et al. [20], the segmental mirroring technique could be exploited satisfactorily for creating reasonably accurate 3D models to enable mandibular reconstruction in instances where preoperative adjustment of a reconstruction plate was constrained by the complexity of the mandibular defect. This strategy was rather less effective when the complicated mandibular condyle anatomy was involved. Similarly, Mahendru et al. [21] advocated the application of CAD/computer-aided manufacturing (CAM) technology and 3D-printed cutting guides to accomplish realistic and cosmetic performance in patients seeking mandibular reconstruction with a free fibula flap. However, the higher costs coupled with CAD/CAM technology can be easily compensated for by reducing the operating time and adequate rehabilitation. Darwich et al. [22] also documented a report of sizeable mandibular tumor removal and reconstruction of the deformity using a personalized 3D-printed titanium implant. The analysis provided impressive functional and aesthetic postoperative outcomes without serious side effects. As per the researcher, titanium implants represented an optimal alloplastic material for mandibular reconstruction since they were versatile, bioinert, impervious, and easily workable, particularly with the aid of 3D digital planning tools. An integrated two-phase strategy was developed by Linares et al. [23] for the segmentation of cone-beam computed tomography (CBCT). They firstly executed an automatic segmentation of bone geometries with super-voxels, permitting a reasonable graph estimate of the 3D data. A user-placed seed operation, then directed a graph partitioning algorithm, which divided the extracted bones into mandible and cranium. The exploration of the algorithm led to a precise segmentation and was resilient to parameter variations. The segmentation of the mandible in computer tomography (CT) scans is greatly affected by metal objects such as dental filling materials and by substantial variations in shape and size between patients. To resolve these challenges, Qiu et al. [24] implemented an automated mandibular segmentation approach in CT scans 
that identified the spectrum of anatomical forms across multiple planes. The mechanism relied on the architecture of the convolution neural network and then merged the resultant two-dimensional (2D) segmentations from three orthogonal planes into a 3D segmentation.

Restoration of maxillary defects following tumor removal is a complex process owing to anatomical and functional complications, and autografts along with fixation plates are amongst the most frequently used methods. The disadvantage of this standard approach is the fairly prolonged time required in manipulating the plate during the process, which not only increases the surgical time but also induces metal fatigue in the plate. The vulnerability of the conventional fixation plates was resolved by Abbai et al. [25], who fused stereolithography with mirror reconstruction through the projection of the unaffected side onto the affected side. Mirroring is one of the most widely used reconstruction techniques, and several research studies proved that the mirror technique successfully restored the facial symmetry [26,27]. Hatamleh et al. [28] also consolidated the mirror restoration method with selective laser sintering for bimaxillary surgery and mandibular reconstruction. The concerned patient reported the capabilities of 3D CAD/CAM technologies in improving surgical results for cranio-maxillofacial reconstruction. Mirror restoration is among the extensively used procedures of 3D reconstruction in medical applications where the impaired section is substituted by a healthy symmetrical region [29]. However, the technique of cavity-filled reconstruction is a new or perhaps uncommon approach where the bone cavity is filled with mass to create a solid bone portion. Since, implant fitting has always depended on the reconstructed area, the accuracy assessment of the restored region is mandatory. This implies that, with better accuracy or placement of the implant, the overall aesthetics of the facial anatomy would improve. The primary goal of this work was to assess the effectiveness of the cavity-filled approach in terms of implant fitting accuracy. It also aimed to compare the performance of the cavity-filled technique with respect to the mirror reconstruction method. For statistical inference, two hypotheses were tested in this work: the null hypothesis of no effect and the alternative hypothesis of an effect [30,31]. The null hypothesis included that there was no difference in the accuracy of the two reconstruction techniques. The alternative hypothesis was that the accuracy of the implant using the cavity-filled approach was significantly different than the mirror reconstruction technique.

In this research, the two techniques (i.e., mirror and cavity-filled reconstruction techniques) were firstly employed in 3D model design and recreation of the implants. The two reconstructed design models were subsequently fabricated in a fused deposition technology (FDM)-based 3D-printing machine (Stratasys, Eden Prairie, MN, USA). These implant models were then evaluated and compared using a $3 \mathrm{D}$ comparison technique. The $3 \mathrm{D}$ comparison is one of the most thorough and useful techniques for quantifying the error deviations between the two models [32]. The accuracy analysis using a 3D comparison was performed in Geomagics Control ${ }^{\circledR}$ software, employing the Faro arm scanner. The two hypotheses were also tested depending on the acquired results. Finally, the implant acquired with the cavity-filled reconstruction approach was fabricated utilizing an electron beam melting (EBM) machine in case the null hypothesis was rejected.

\section{Proposed Methodology}

The proposed research methodology adopted for the reconstructive design consisted of six stages as shown in Figure 1.

\subsection{Tumor Diagnosis}

A patient with a progressive expanding mass on his left cheek was subjected to an incisional biopsy procedure, which showed a plexiform ameloblastoma. A GE (General Electric) light speed VCT XTe 64-row spiral CT scan was used, and the scanned images were then saved as a DICOM (digital imaging and communication in medicine) file. DICOM is a de facto international standard for sharing, exchanging, and storing digital medical images. The DICOM file contained 2D images of the anatomical structure. 


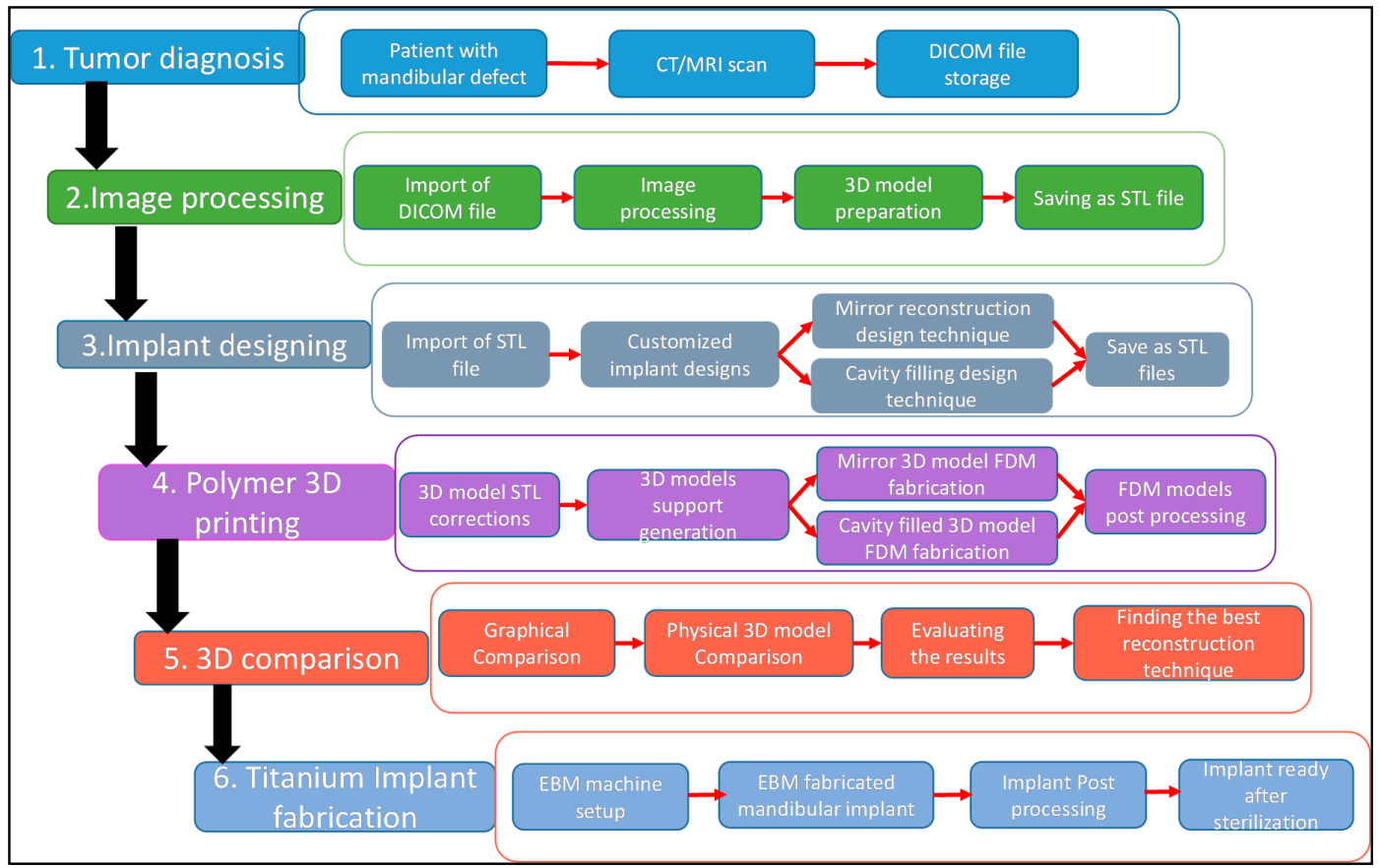

Figure 1. Flowchart illustrating the steps in the selection of implant reconstruction technique.

\subsection{Image Acquisition and Processing}

The DICOM file consists of a series of 2D images that do not provide enough information for clinical diagnosis. There are many image visualizations and surgical planning software available in the market such as 3D Slicer, Scan IP, etc. In this study, MIMICS ${ }^{\circledR} 17.0$ (Materialise NV, Leuven, Belgium, 2014), a powerful image processing software for 3D design and modeling, was utilized in the conversion of medical DICOM data into a 3D surface model. In MIMICS ${ }^{\circledR}$, the 2D DICOM images were stacked upon each other to create a perfect 3D surface model, as shown in Figure 2. The 3D model showed a cystic cavity with a cortical bone defect in the left mandibular body region.

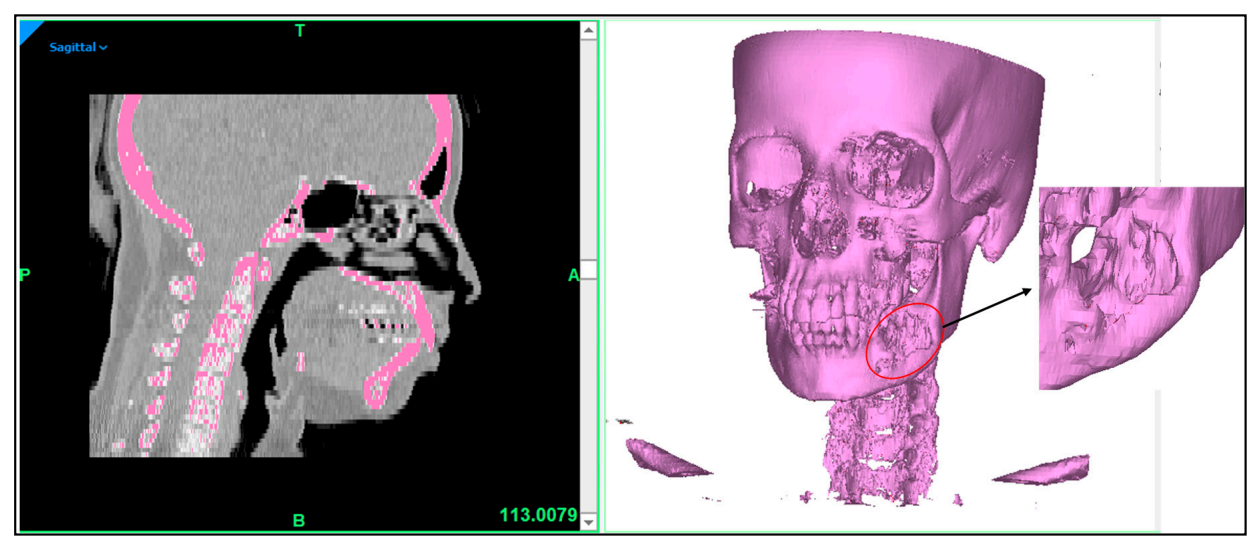

Figure 2. Patient anatomical model revealing the tumor mass and the resultant bone destruction on the left side of the mandible.

A custom Hounsfield unit (HU) of upper threshold value 3012 and a lower threshold value of 260 was used for the bone identification and to separate the hard and soft tissues. The next step was to segment the full mask into a series of smaller elements using a region-growing technique, until the region of interest (mandible) was obtained (Figure 3). The surface irregularities were smoothened 
with a compensating shrinkage factor and the obtained mandible was then saved as an STL (Standard Triangle Language) file.

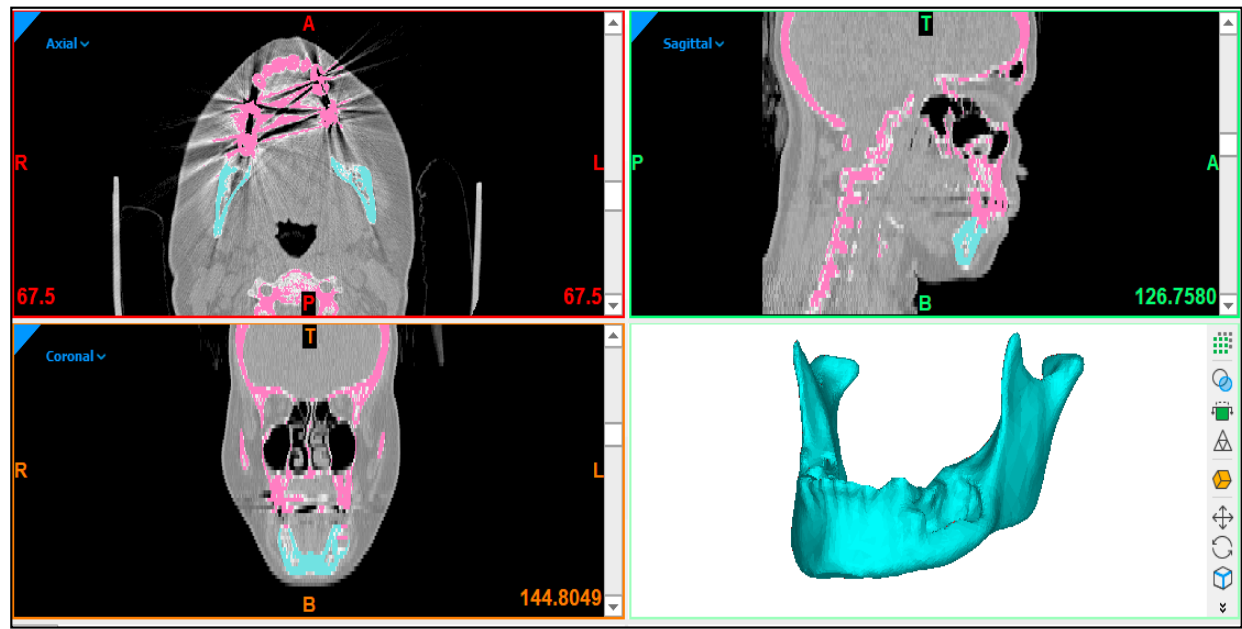

Figure 3. Segmentation of the region of interest (mandible) from the skull region.

\subsection{Custom Implant Design}

The mandible model from MIMICS ${ }^{\circledR}$ was imported in 3-Matic ${ }^{\circledR} 13.0$ (Materialise NV, Leuven, Belgium, 2018) software to model and custom-design the reconstruction plate. The steps involved in mirror reconstruction are explained in Figure 4. The mandibular STL file obtained from MIMICS ${ }^{\circledR}$ was imported in 3-Matic ${ }^{\circledR}$ (Figure 4a), where the left tumor region was cut down by a cut-and-punch operation (Figure 4b). The mandibular model was then split into two regions (Figure 4c), where the tumor region was removed (Figure 4d) for mirror operation. Mirroring was performed (Figure 4e) where the symmetrical healthy right mandibular region was mirrored to the left side. The two symmetrical right and left mandibular regions were then merged (Figure 4f) and wrapped to remove the voids and gaps (Figure 4g). The final obtained mirror model was then aligned with the mandibular tumor model (Figure $4 \mathrm{~h}$ ) to evaluate the anatomical positions. Upon closer examination of the aligned models, a small upper portion of the condyle regions mismatched the original tumor mandible. Several attempts at re-mirroring and re-adjustment failed to match the orientation to the original mandible.

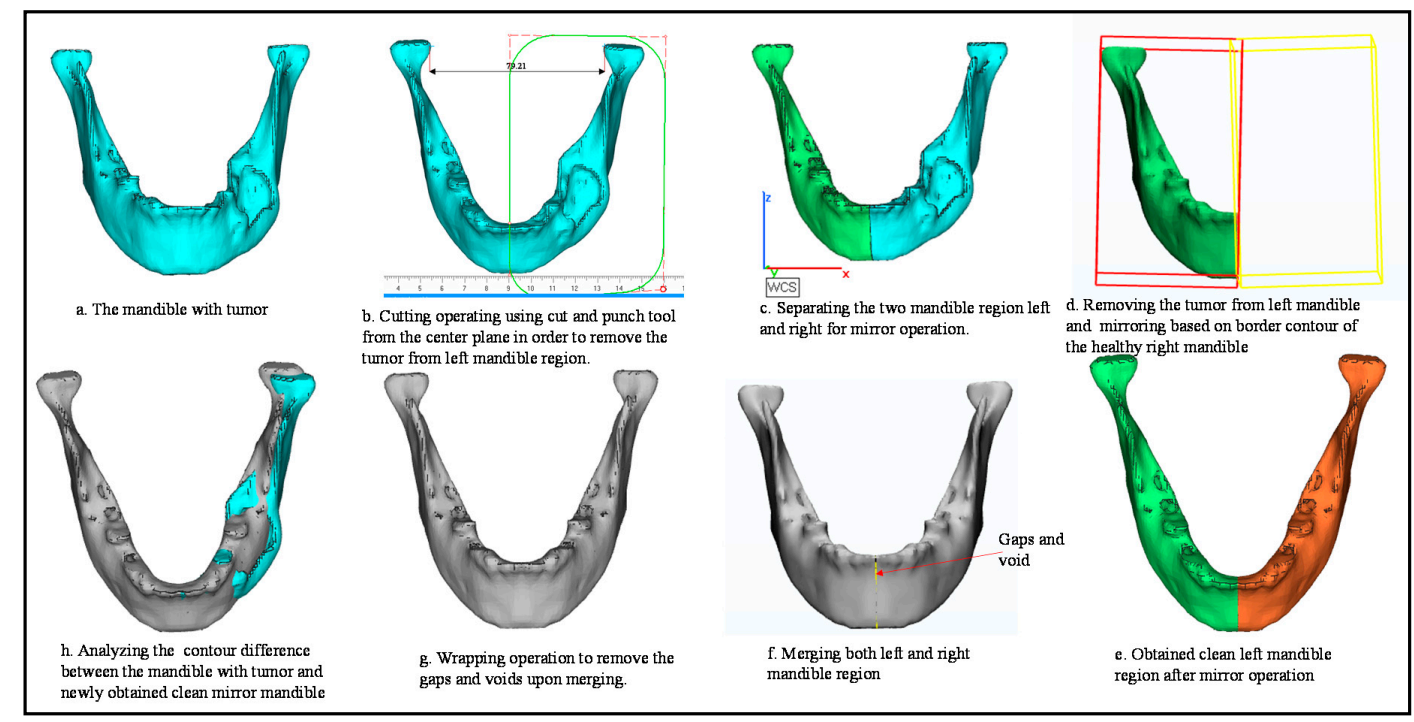

Figure 4. Steps involved in the three-dimensional reconstruction of the clean, healthy mandible using the mirroring technique. 
To overcome the misalignment between the mirroring and original tumor mandible, a newly design reconstruction technique was proposed. In this technique, the tumor model (Figure 5a) was cavity-filled to create a solid bone portion matching the original contours (Figure 5b). The benefit of such an approach was that it maintained the shape and anatomical geometry of the mandible. The additional bone portion of the tumor area was trimmed (Figure 5c), and the obtained cavity-filled mandible model was then aligned with the tumor mandible for evaluation (Figure 5d).

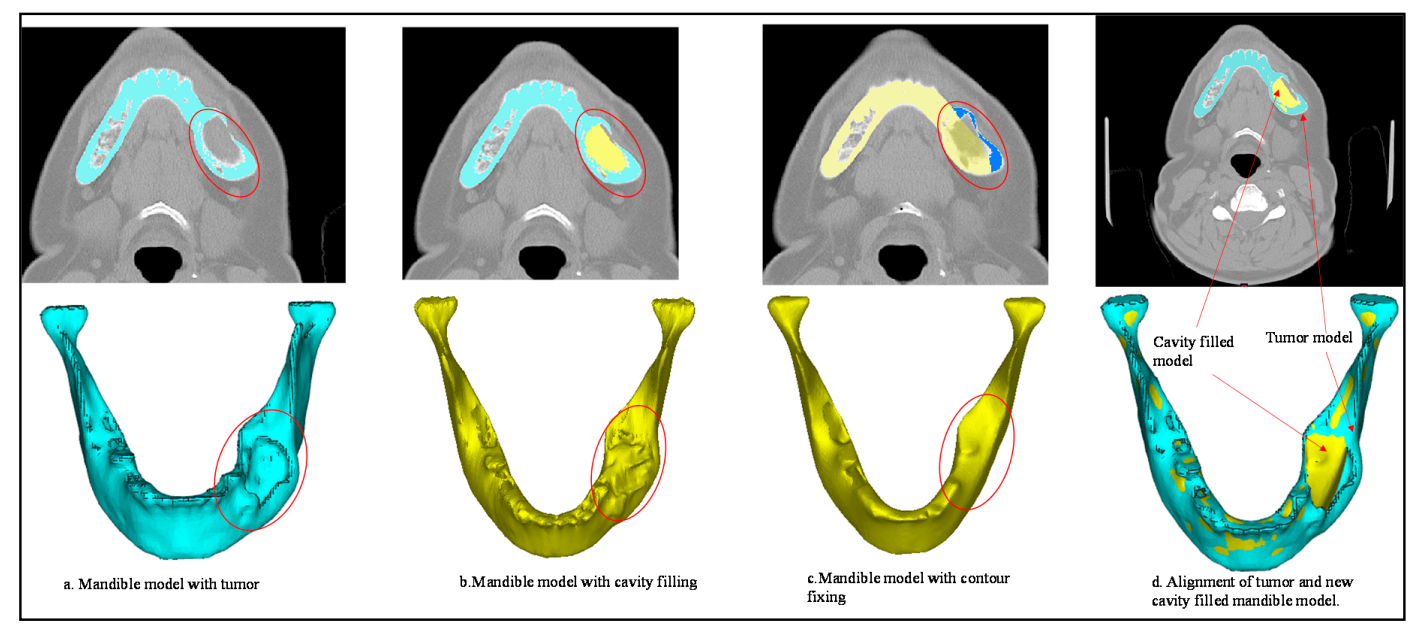

Figure 5. Sequential steps involved in the reconstruction of the mandible using cavity-filled technique.

Figure 6 explains the design steps involved in the resection of the mandible model, where the tumor part (Figure 6a) was resected using a cut-and-punch operation (Figure 6b,c). The resected model was the patient's mandible bony region on which the surgeons operated the implant after tumor removal. The resected tumor mandible (Figure 6d) was used for the 3D comparison and the fitting of the customized implant design in this study.

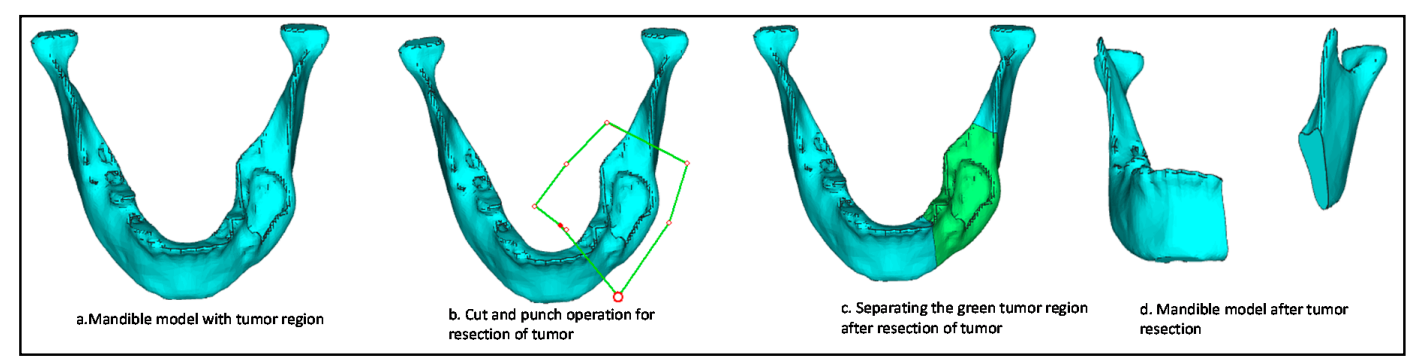

Figure 6. Design steps in the creation of resected mandible.

Figure 7 explains the steps involved in the reconstruction of the customized implant design from the cavity-filled model. The obtained cavity-filled mandible model was loaded into 3-Matic ${ }^{\circledR}$ as an STL file (Figure 7a). The model outer part was selected using the brush mark technique (Figure $7 \mathrm{~b}, \mathrm{c}$ ) and extracted for custom implant design (Figure $7 \mathrm{~d}$ ). Trimming and splitting operations were performed (Figure 7e,f) on the extracted bone mask to get an implant template (Figure $7 \mathrm{~g}$ ). An offset thickness of $2 \mathrm{~mm}$ was added (Figure 7h), and screw holes were incorporated for fitting (Figure 7i). The final obtained prefabricated custom-designed mandibular implant was aligned with the resected mandible for assembly and fitting evaluation (Figure 7j). The prefabricated implant designed from the cavity-filled model fitted precisely onto the tumor-resected mandible model as it was created from the bone itself. Similarly, a second prefabricated implant was also designed following a similar approach to that above from the mirror reconstruction model. These steps are presented to illustrate how the custom-designed implant was created from the healthy mandible after tumor removal through 
a mirroring or cavity-filled approach. Hence, reconstructing a healthy mandible model is important whether through mirroring or cavity-filled approaches. The modeling and designing of the customized implant from the healthy mandible is the same for both models.

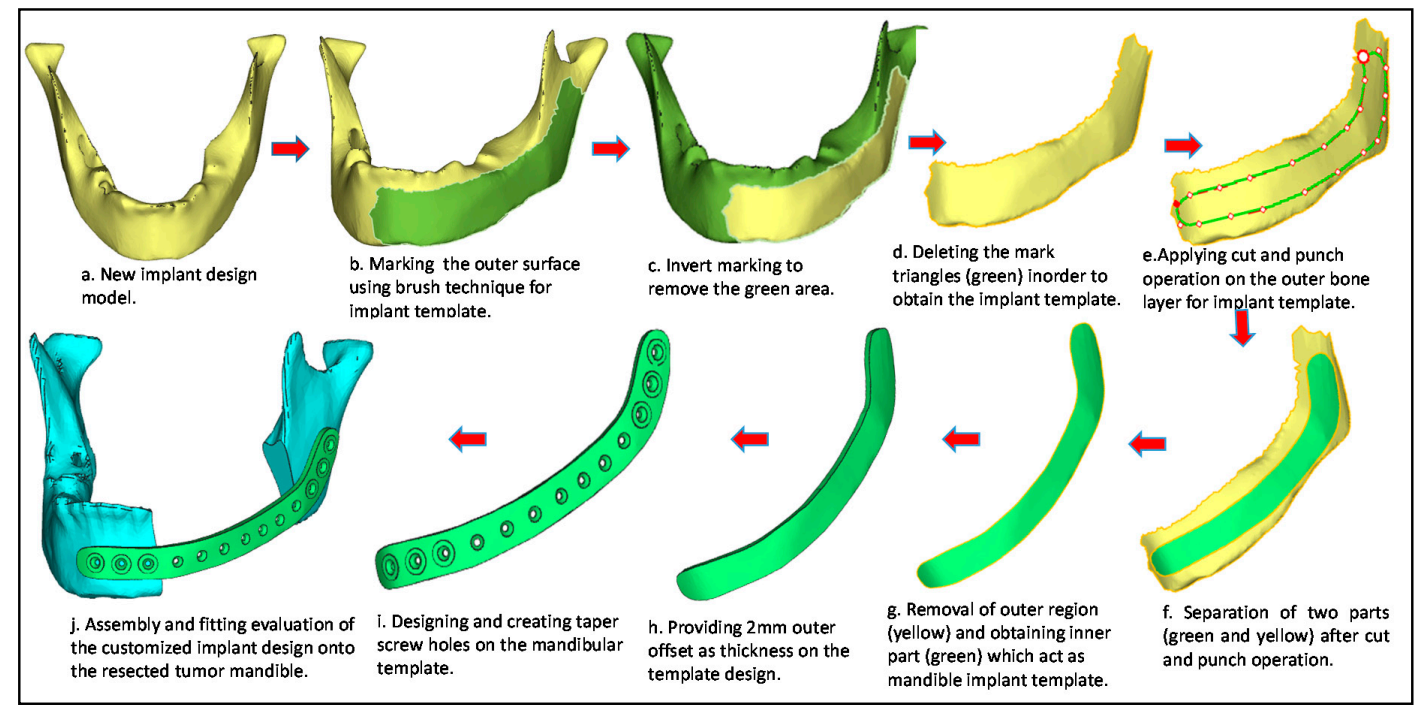

Figure 7. Steps involved in the design of a customized mandibular implant from a healthy design model.

\section{3D Printing}

The technique of 3D printing was used in this study for the fabrication of the mandibles and the subsequent $3 \mathrm{D}$ comparison of the reconstructed design models. The polymer-based Stratasys FDM machine was used for the 3D printing of models. The FDM machine used ABS (acrylonitrile butadiene styrene) material, which is a common thermoplastic resin allowing functional testing of medical products, and which is relatively safe to handle [33]. The ABS material contributes a good balance of chemical and heat resistance, along with tensile strength and a good glossy finish on the finished surface [34]. The FDM process started by importing the model STL file into a pre-processing software where the model was sliced into a series of horizontal 2D layers. Support structures were created where needed as per the model geometry. The built and support filaments were unwound from a coil and fed through the printer extrusion nozzle. The nozzle melted the filament and extruded it on top of the previous extrusion layer, thus creating parts layer by layer.

\subsection{STL Corrections and Support Generations}

The mirror and cavity-filled reconstructed models were firstly subjected to Magics ${ }^{\circledR} 18.0$ (Materialise NV, Leuven, Belgium, 2013) to repair the STL file and to generate support. Repairing the STL file before 3D printing was a mandatory operation, because any faulty STL file could lead to geometric inaccuracies and a defective build [35]. Support structures were used to dissipate the heat and to assist in overhanging structures [36]. Moreover, Magics ${ }^{\circledR}$ was also used for the orientation and best possible position during the build process to minimize the build time for the support structure.

\subsection{FDM Fabrication}

The error-free STL files of the reconstruction models were imported into the FDM machine for the fabrication of polymer mandibular models. Figure 8 illustrates the FDM machine and its fabricated original resected mandible with support structures. 


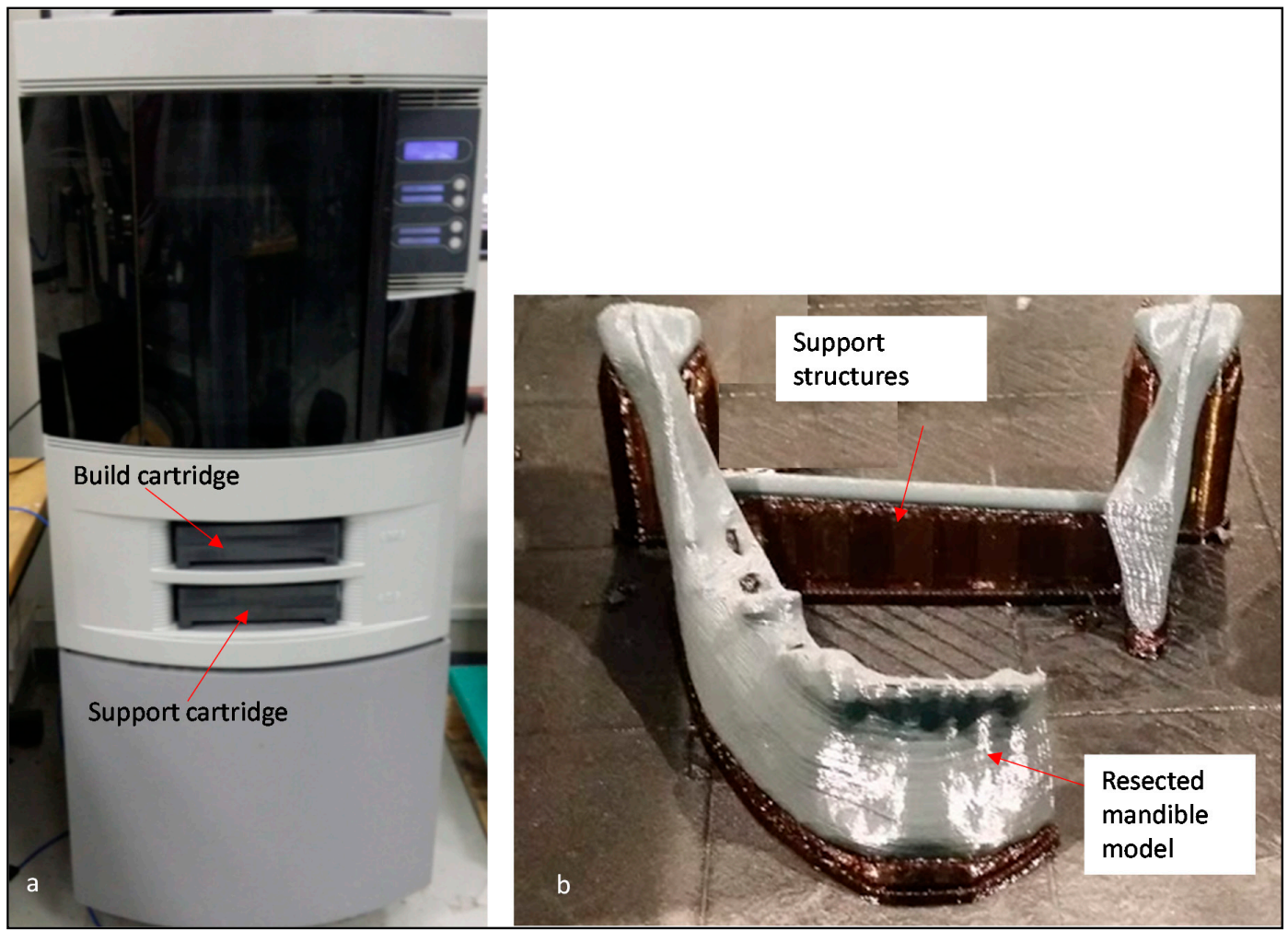

Figure 8. Fused deposition modeling (FDM) machine (a) with built resected mandible model, and (b) with support structures.

The supports were removed by releasing the fabricated parts into a heated water-based soluble solution where the support structure was dissolved. Figure 9 illustrates the final obtained FDM fabricated models after support removal.

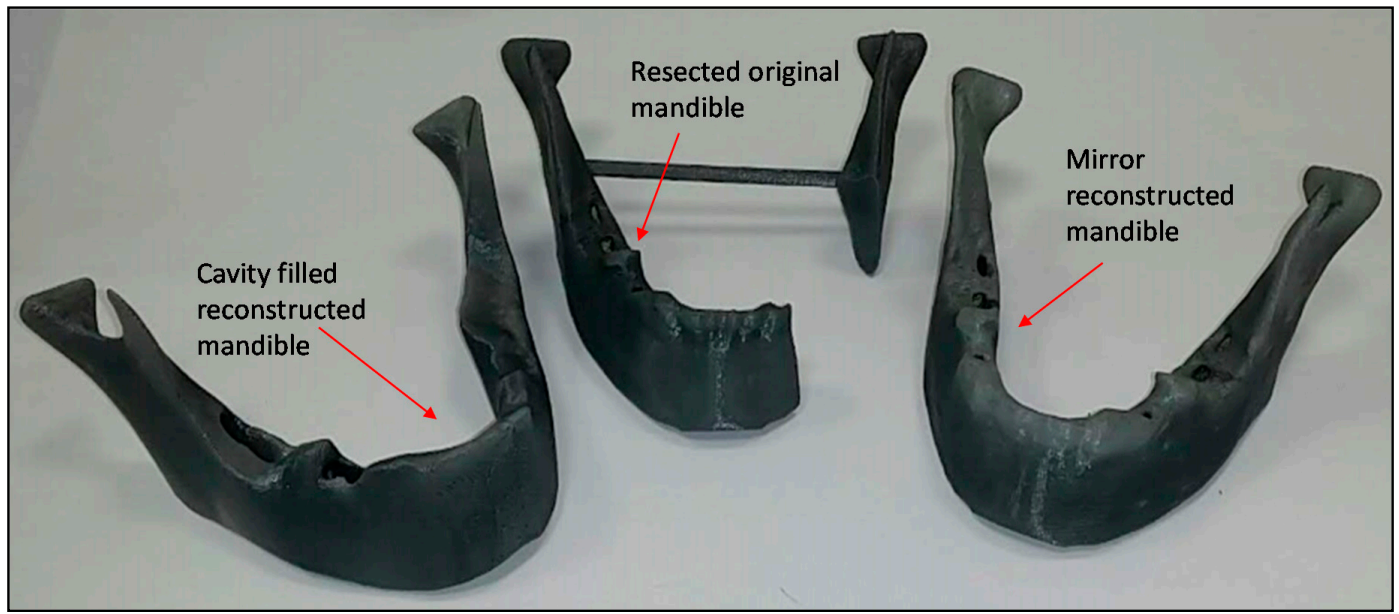

Figure 9. FDM-fabricated polymer models: cavity-filled reconstructed model (right), resected original model (center), and mirror reconstructed model (left) after support removal.

\section{Accuracy Assessment}

Convenient and precise fitting of the implant is necessary for an aesthetic and functional mandibular restoration. The accuracy of the implant fitting is invaluable, especially when a defect is present on facial features, such as the mandible. The implant fitting is indeed dependent on the reconstructed area obtained via mirroring and cavity-filled techniques. The restoration of the defective 
area for implant design requires several phases from data acquisition to eventual fabrication. Certainly, errors can occur at each step, and the final accuracy is the sum of all errors at each stage. Therefore, accuracy assessment is mandatory to enhance the placement of the implant and the overall aesthetics of the facial anatomy.

The accuracy evaluation in this investigation was based on a comparison between the resected and the reconstructed mandible models. This analysis was conducted in two steps, to validate and corroborate the findings. Firstly, the STL files of the resected and recovered mandibles were compared, and the polymer-based prototypes were examined in a subsequent stage to achieve a precise fitting of the implants. The goal of this step was, therefore, to estimate the accuracy or precision of the defect reconstruction methods in partially edentulous patients by superimposing 3D digital (STL) files, as well as physical prototypes. The overall workflow for accuracy evaluation is shown in Figure 10.

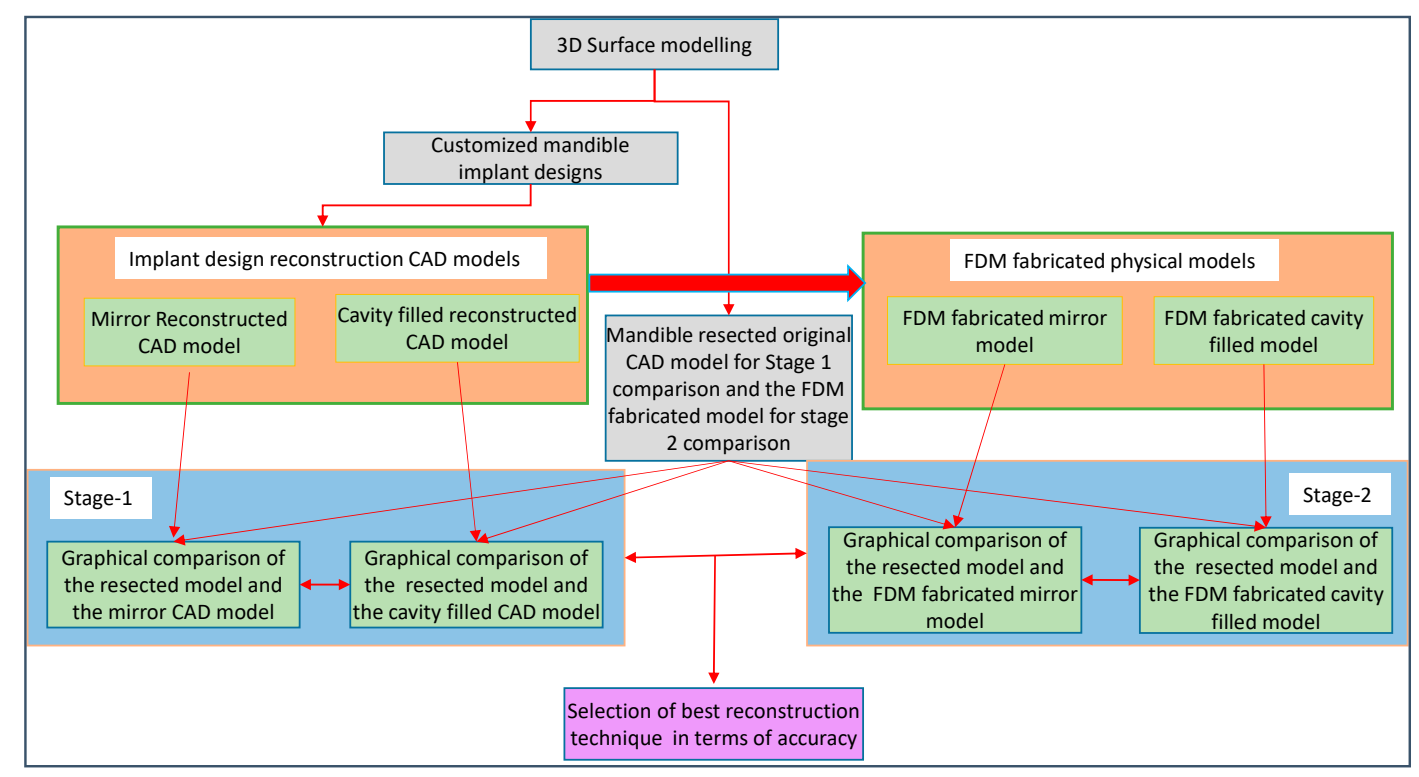

Figure 10. Evaluation of an implant design reconstruction technique using a comparison approach.

For assessment based on physical prototypes, the FDM-produced mirroring and cavity-filled models, as well as the resected model, were scanned using the FARO arm laser scanner (Faro, Lake Mary, FL, USA), as shown in Figure 11. The obtained files were imported into the Geomagics Control 2014 software (3D Systems, Rock Hill, SC, USA) for analysis. For STL-based analysis, the STL files of mirroring, cavity-filled, and resected models were directly imported into the Geomagics Control software from MIMICS. To analyze the deviation (or accuracy), the STL files of the resected mandible were superimposed one by one on both mirror and cavity-filled mandibles via perfect matching using the best-fit algorithm. The resected model was set as a reference, while the mirrored and cavity-filled models were set as test models to facilitate the accuracy assessment.

The following 3D deviations were measured for accuracy analysis using Geomagics Control: root-mean square (RMS) and average deviation (AD). The AD required estimating the variations in the inside and outward directions between the test and the reference files. The RMS (Equation (1)) determined the average error magnitude between both datasets or models. It also quantified the overall accuracy of the models. The two statistics were considered to ascertain and substantiate the results.

$$
\mathrm{RMS}=\frac{1}{\sqrt{n}} \sqrt{\sum_{i=1}^{n}\left(X_{1, i}-X_{2, i}\right)^{2}}
$$


where $X_{1, i}$ is the $i$-th digitized point in the reference dataset, $X_{2, i}$ is the $i$-th digitized point in the test dataset, and $n$ represents the number of digitized points. The units of measurement considered for the RMS and AD were mm.

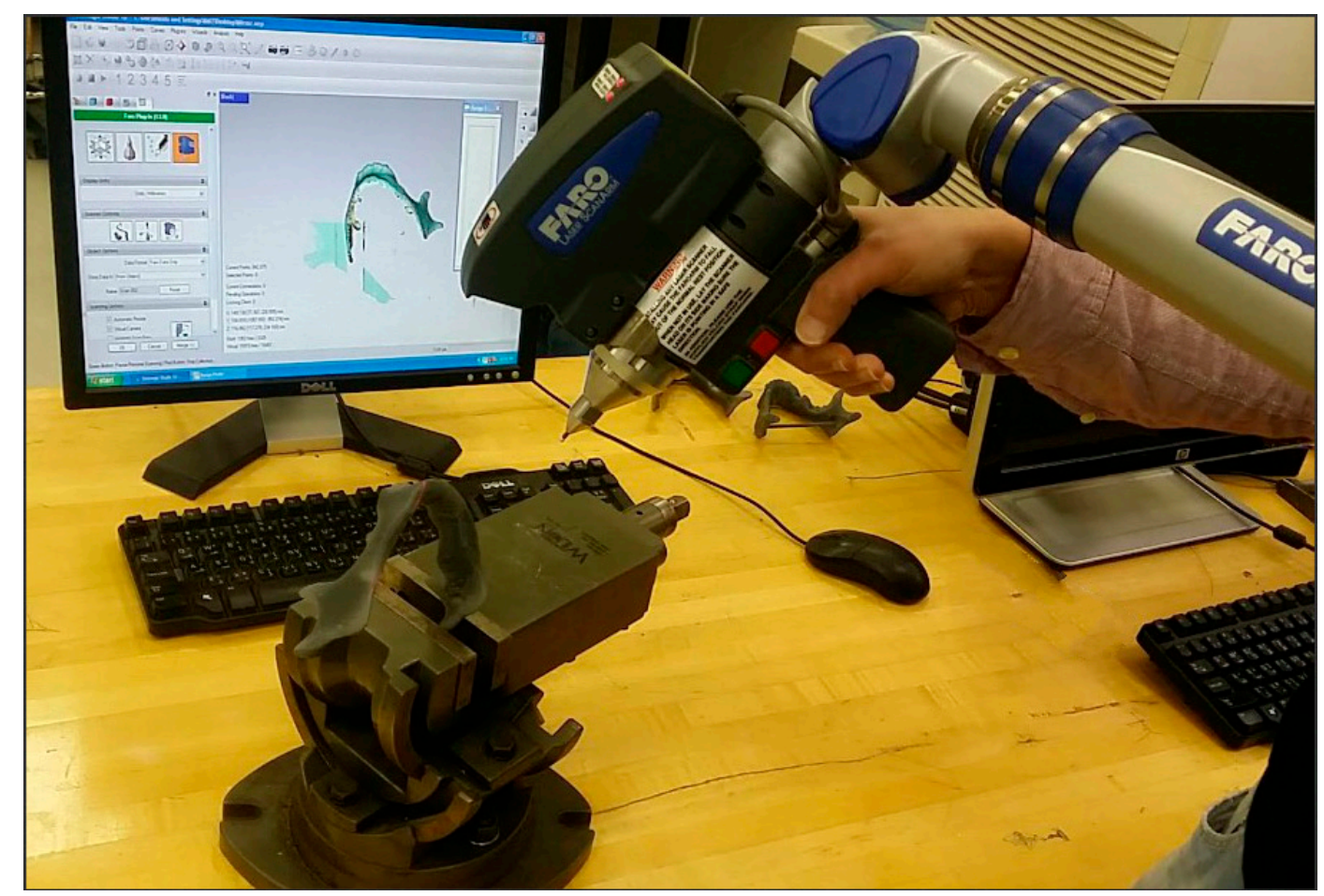

Figure 11. Data acquisition from FDM-reconstructed models using a FARO arm laser scanner.

\section{Results}

The 3D inspection results shown in Figure 12 illustrated that the majority of the surface was mapped green in the cavity-filled approach, suggesting a high degree of precision between reference (R) and test (T) models.
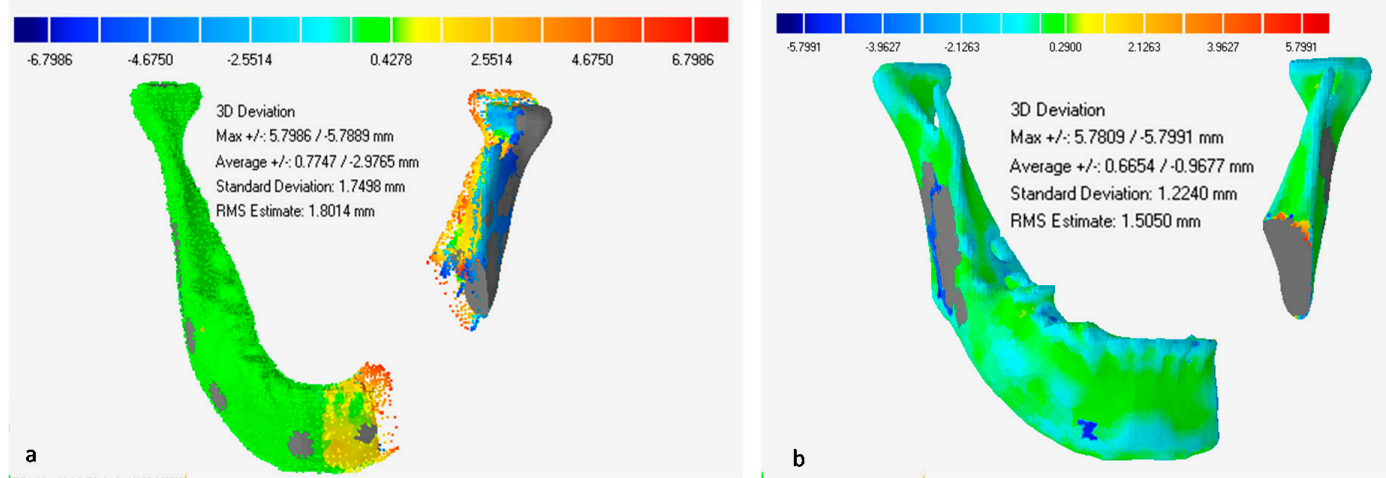

Figure 12. Three-dimensional (3D) inspection results of the resected original mandible model with

(a) mirror reconstruction model and (b) cavity-filled reconstruction model.

A 2D analysis was also conducted to determine the variance around a specific cross-section. A 2D comparison is useful for matching the cross-section of the test object to the cross-section of the reference component. The section plane, shown in Figure 13, was taken across the middle of the mandible to divide the part into top and bottom halves. Figure 13a implies that the top half of the mandible in the test portion was not precisely aligned with the reference artifact. This could be due to either inaccurate 
symmetrical measurement of the plane or differences on both sides of the mandible of the patient. Indeed, the 2D analysis in Figure 13b demonstrated the higher precision of the cavity-filled approach as compared to the mirror-reconstructed mandible.

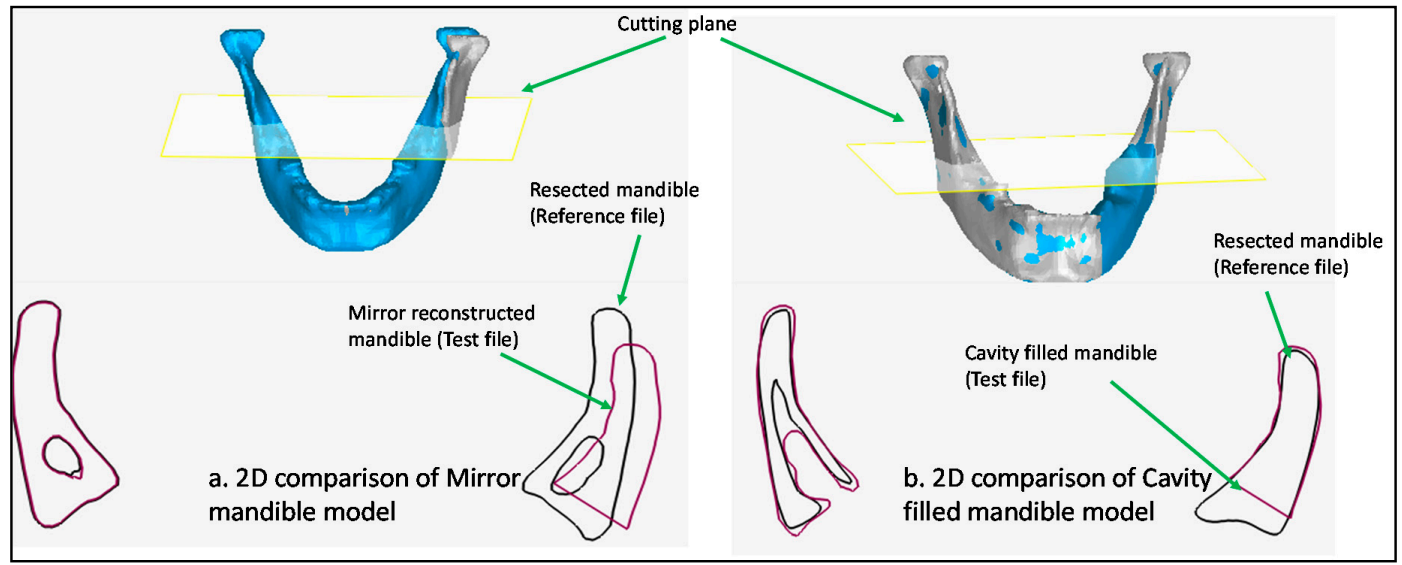

Figure 13. Two-dimensional (2D) comparison results of the cross-sectional plane of resected mandible with (a) mirror mandible model and (b) cavity-filled model.

The findings of the STL file comparison were further assisted by examining the FDM-produced physical prototype scans using the Faro arm laser scanner. The 3D color map, shown in Figure 14a, further determined that the cavity-filled reconstruction technique resulted in higher accuracy.
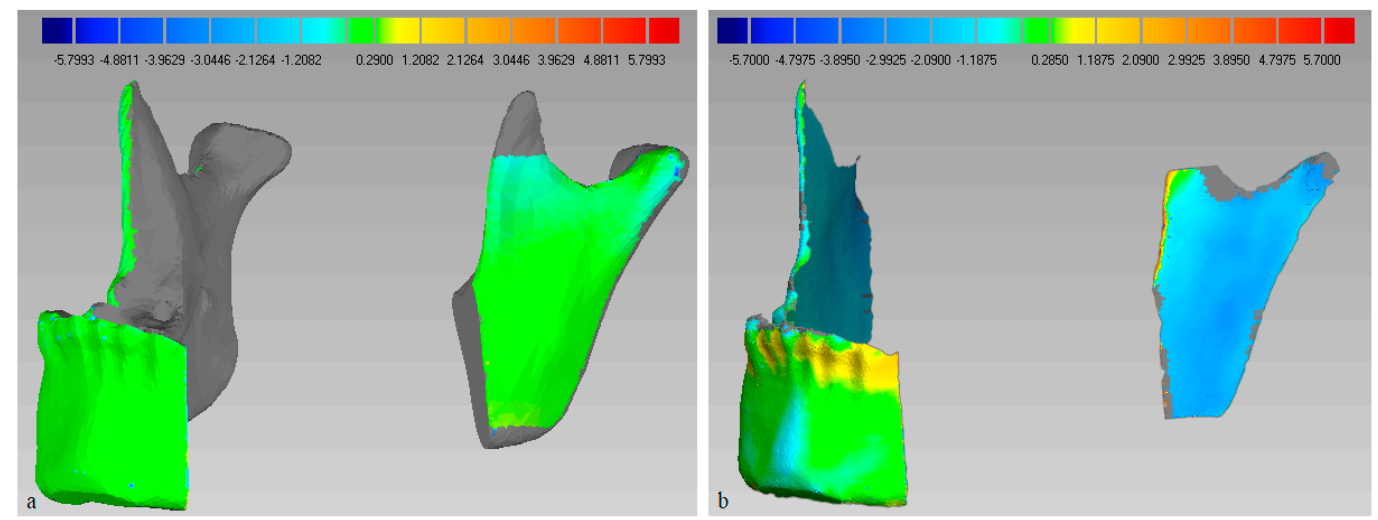

Figure 14. The 3D comparison results for physical prototypes with (a) Cavity-filled with resected comparison (b) Mirror with resected comparison.

The Geomagics program produced the statistical information, including the RMS and AD (positive and negative sides), upon alignment of the two models. There was a positive deviation when the test model was outside the reference model and conversely for a negative deviation. A series of five measurements was conducted for each mode under study to validate the consistency of the method, as shown in Table 1. The placement and positioning of the specimen or mandible was altered at each measurement run. A comparative analysis of the mirror and cavity-filled techniques with respect to their accuracy (RMS and AD) was made using the two sample $t$-test. As the $p$-value was less than the significance level $(\alpha)$ of 0.05 , the null hypothesis was discarded, and it could be inferred that the two reconstruction techniques differed significantly.

Furthermore, the one-way ANOVA test also verified that the $p$-values for the RMS and AD between mirror and cavity-filled groups were less than 0.05 . This finding substantiated yet again that both techniques (mirror and cavity-filled) varied considerably. The outcomes of both the sample $t$-test 
and the one-way ANOVA led to the rejection of the null hypothesis and concluded that the population means from two reconstruction techniques were not equal.

The mean RMS values observed for mirroring and cavity-filled techniques in the physical prototype evaluation were 1.2683 and $1.1019 \mathrm{~mm}$, respectively. This implied an error decline of nearly $13 \%$ in 3D-printed mandibles restored using a cavity-filled approach, as depicted in Figure 15. Similarly, when the STL files from the mirror and the cavity-filled rehabilitated virtual variants were assessed, the RMS error value decreased from 1.8018 to $1.5048 \mathrm{~mm}$, resulting in an approximately $16 \%$ drop. The significant decrease in AD (both positive and negative in Table 1) can also be noted in the cavity-filled procedure relative to the mirror reconstruction solutions. This provided mean values of $0.5194 /-0.5392 \mathrm{~mm}(\mathrm{AD})$ for the cavity-filled approach as compared to the mirroring technique, which resulted in corresponding higher values of $0.7441 /-0.8682 \mathrm{~mm}$ (see Table 1 ). This reduction in error is very competitive, given the simplicity and ease of use of the cavity-filled method. The preceded analysis underlines that the cavity-filled method would lead to a more reliable implant that should meet the aesthetic requirements. However, in mirror reconstruction, the approximation of the midsagittal plane is done using manual landmark points, which leads to deviation and causes implant placement issues, as well as concerns in post-surgical rehabilitation and movement. In contrast, cavity-filled reconstruction is a rather more straightforward and robust technique without any landmarks.

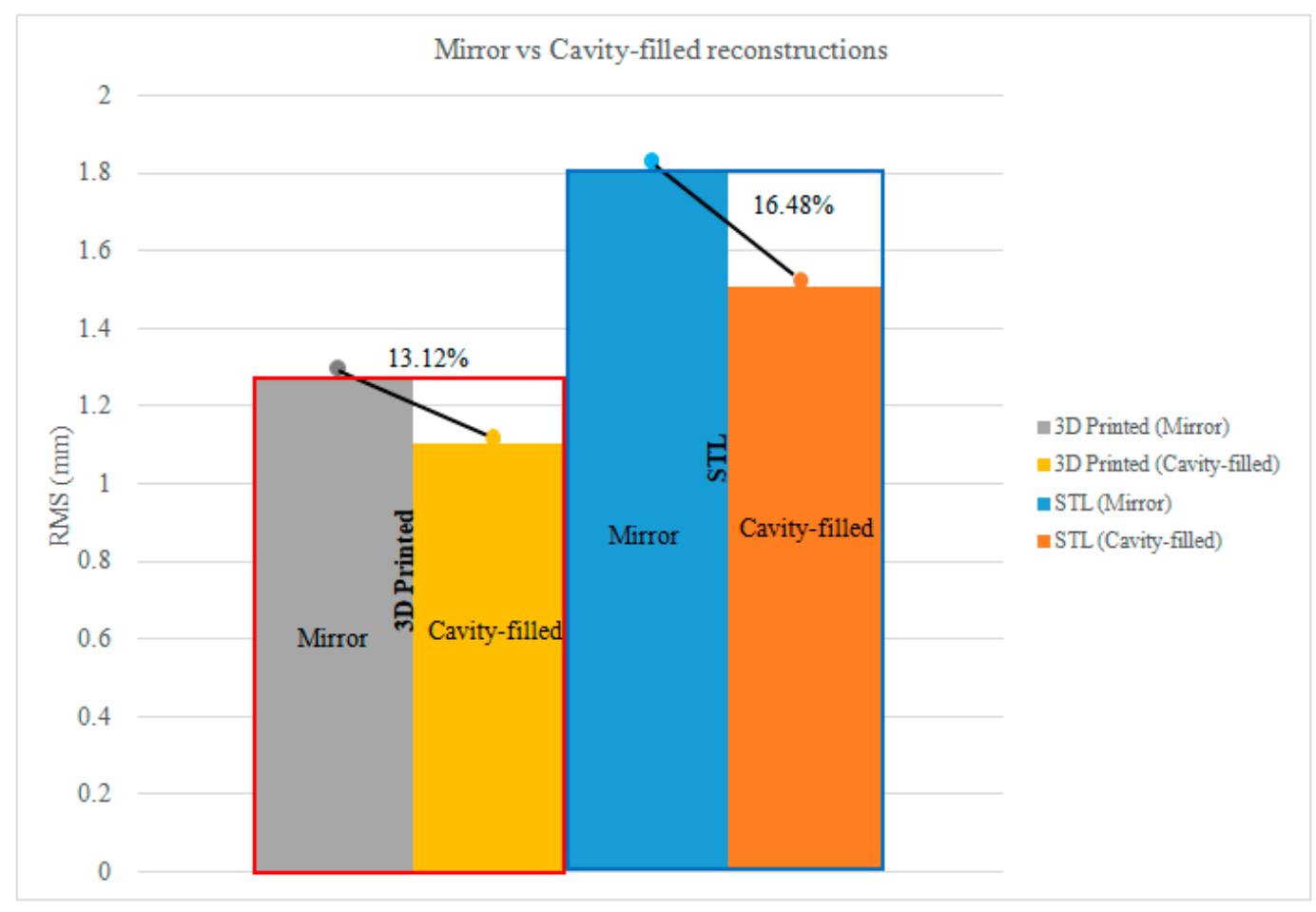

Figure 15. Graphical depiction of error reduction with cavity-filled approach. 
Table 1. The 3D comparison results of the mirror and the cavity-filled reconstruction techniques.

\begin{tabular}{|c|c|c|c|c|c|c|c|c|c|c|c|c|}
\hline \multirow{4}{*}{$\begin{array}{l}\text { Sample } \\
\text { Number }\end{array}$} & \multicolumn{12}{|c|}{ Mode (R-T) } \\
\hline & \multicolumn{3}{|c|}{ Scanned (Resected)- Scanned (Mirror) } & \multicolumn{3}{|c|}{ STL (Resected)- STL (Mirror) } & \multicolumn{3}{|c|}{ Scanned (Resected)- Scanned (Cavity-Filled) } & \multicolumn{3}{|c|}{ STL (Resected)- STL (Cavity-Filled) } \\
\hline & \multirow{2}{*}{ RMS } & \multicolumn{2}{|c|}{ Average } & \multirow{2}{*}{ RMS } & \multicolumn{2}{|c|}{ Average } & \multirow{2}{*}{ RMS } & \multicolumn{2}{|c|}{ Average } & \multirow{2}{*}{ RMS } & \multicolumn{2}{|c|}{ Average } \\
\hline & & Negative & Positive & & Negative & Positive & & Negative & Positive & & Negative & Positive \\
\hline 1 & 1.2695 & -0.8676 & 0.7432 & 1.8014 & -2.9765 & 0.7747 & 1.1021 & -0.5386 & 0.5192 & 1.5050 & -0.9677 & 0.6654 \\
\hline 2 & 1.2690 & -0.8672 & 0.7434 & 1.8017 & -2.9760 & 0.7753 & 1.1011 & -0.5390 & 0.5198 & 1.5047 & -0.9680 & 0.6650 \\
\hline 3 & 1.2672 & -0.8693 & 0.7432 & 1.8031 & -2.9762 & 0.7730 & 1.1014 & -0.5397 & 0.5196 & 1.5054 & -0.9671 & 0.6655 \\
\hline 4 & 1.2664 & -0.8690 & 0.7424 & 1.8025 & -2.9769 & 0.7742 & 1.1023 & -0.5389 & 0.5193 & 1.5046 & -0.9670 & 0.6652 \\
\hline 5 & 1.2692 & -0.8678 & 0.7483 & 1.8004 & -2.9779 & 0.7758 & 1.1028 & -0.5399 & 0.5190 & 1.5044 & -0.9679 & 0.6649 \\
\hline Mean & 1.2683 & -0.8682 & 0.7441 & 1.8018 & -2.9767 & 0.7746 & 1.1019 & -0.5392 & 0.5194 & 1.5048 & -0.9675 & 0.6652 \\
\hline
\end{tabular}




\section{EBM Fabrication}

The finally obtained cavity-filled implant was produced through EBM technology. EBM is one of the latest rapid manufacturing techniques, which produces a fully dense part using a computer-controlled gun in a vacuum environment. EBM was first commercialized in 1997 by the Arcam AB Corporation now under General Electric [37]. The general principle of EBM is the selective melting of metal powder (Ti6Al4V) in a layer-by-layer fashion using the electron beam of an electron emitted from the tungsten filament. The beam of electrons, as illustrated in Figure 16a, is controlled by three magnetic lenses, namely, the astigmatism lens, the focus lens, and the deflection lens. The astigmatism lens helps to generate a circular electron beam with a Gaussian energy distribution. The focus lens focuses and sharpens the beam into the smallest desired $(0.1 \mathrm{~mm})$ diameter. The deflection lens deflects the electron beam onto a specific target of the powder bed as per the CAD file. When the high-energy beam of electrons hits the metal powder, the kinetic energy is transformed into heat and melts the powder completely. After each melt cycle, the powder hopper holding the metal powder of 45 to 100 microns in size (Figure 16b) is lowered by one layer (50 microns), and the next layer of powder is fed onto the build platform using raking blades. This process is repeated until the completion of the build.

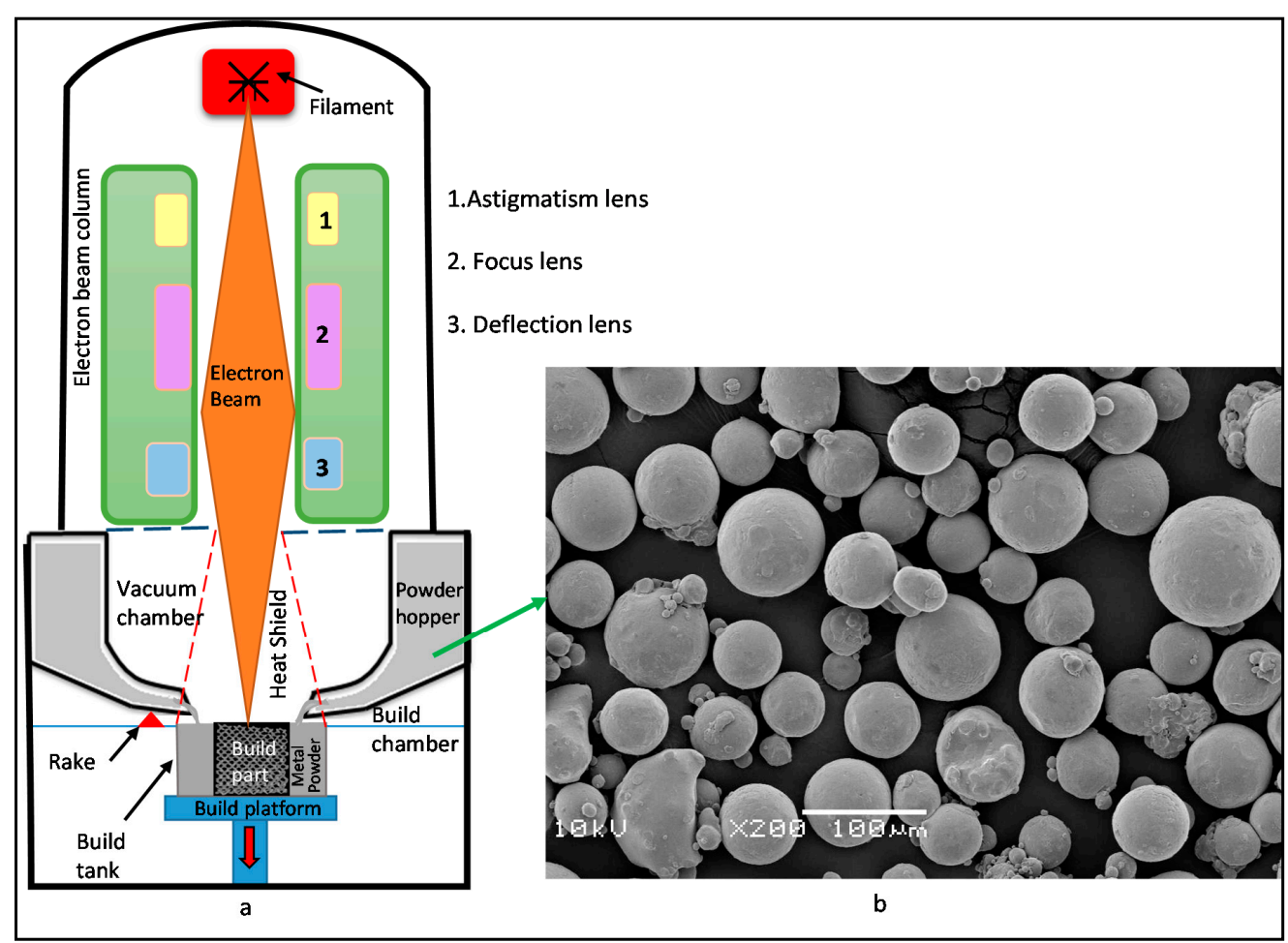

Figure 16. (a) Schematic diagram of the electron beam melting (EBM) A2 machine and (b) scanning electron microscopic image of Ti6Al4V powder.

When the build was completed, the build chamber was allowed to cool to $100{ }^{\circ} \mathrm{C}$ at $400 \mathrm{mbar}$ through helium, followed by air. The cooled EBM build parts were then fed into a powder recovery system (PRS) (Figure 17) to blast away the semi-sintered titanium powder attached to the building part. All the blasted powder was recycled to minimize material loss. A filter screen and explosion-protected vacuum cleaner was also used to separate the coarse powder particles from the clean powder.

Figure 18a illustrates the EBM-fabricated cavity-filled mandibular implant with attached support structures. The support structures provided gravity support for overhanging surfaces in order to dissipate the process heat and to minimize the geometrical distortions caused by the internal stresses [38]. However, the support structures had to be removed during post-processing. The support structures were attached to the building part by the teeth (Figure 18b) which could be easily removed 
manually through plyers. The finally obtained titanium implant after support removal was precisely fitted onto the mandible model using bi-cortical medical screws, as shown in Figure 18c,d. This precise adaptation of the implant and bone counterpart helps in the reduction of surgical time and revision, in addition to providing better cosmesis [39].

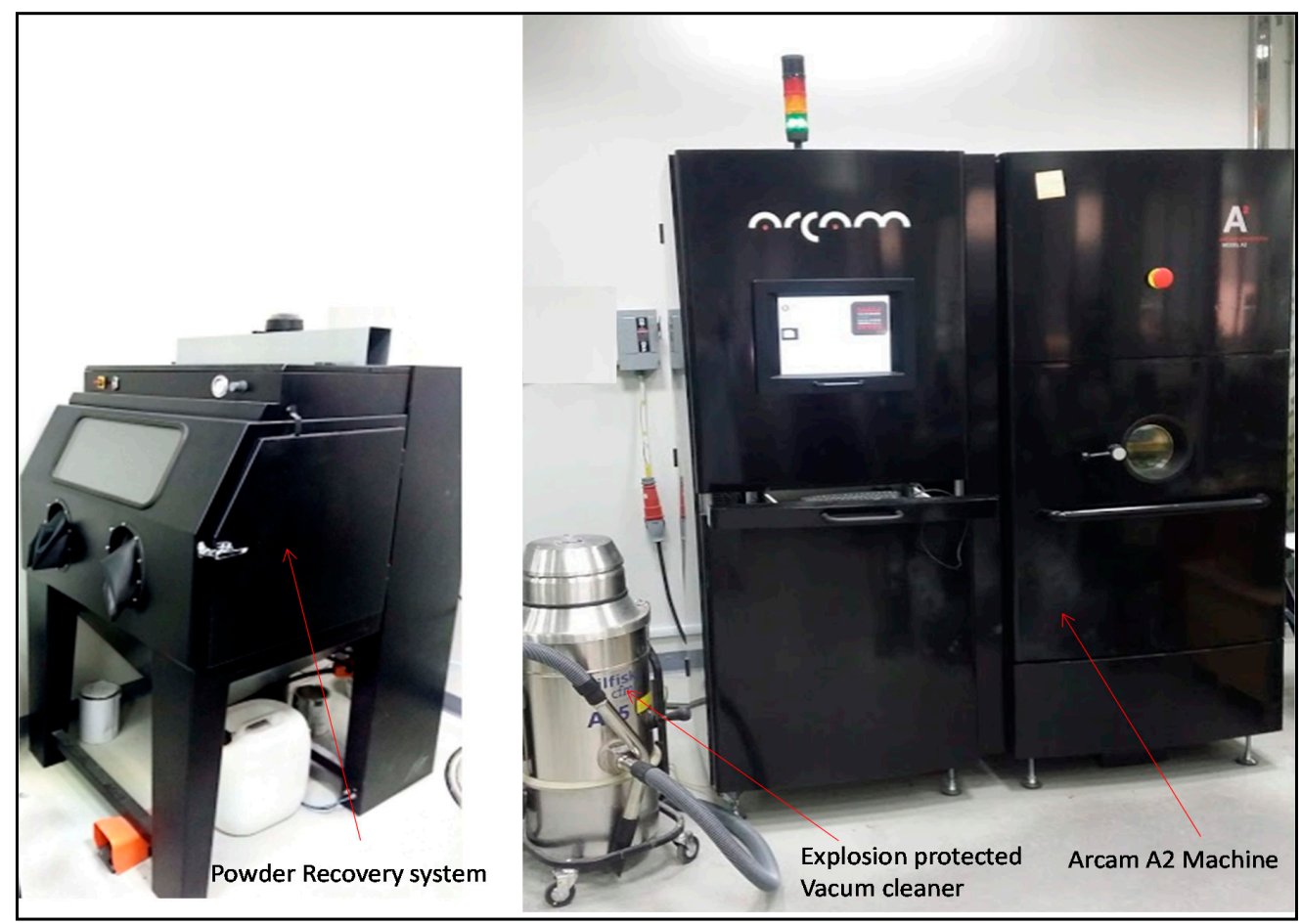

Figure 17. An Arcam EBM A2 machine (ARCAM AB, Molndal, Sweden) with accessories such as a powder recovery system and explosion-protected vacuum cleaner.
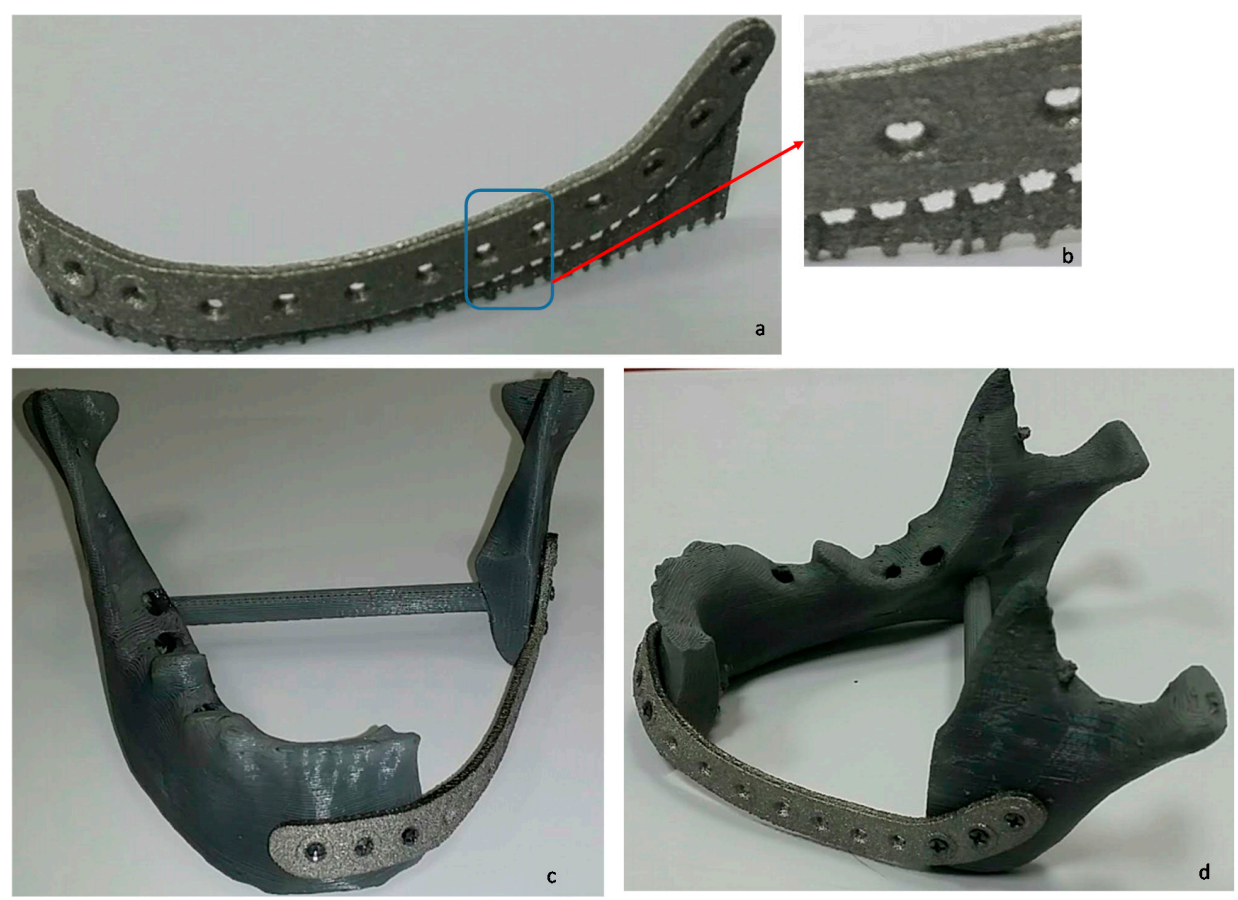

Figure 18. EBM-produced cavity-filled reconstructed titanium implant (a) with support structures attached to the teeth (b) for easy removal. Front view (c) and side view (d) of precisely fitted titanium implant onto the FDM model with bi-cortical screws after support removal. 


\section{Discussion}

This work illustrated the significance of effective and accurate reconstruction techniques for accomplishing enhanced aesthetic and functional needs of a mandible. This is because the mandible and maxilla plays an important role in mastication, articulation, and cosmetic functions. They are both amenable to composite bone reconstruction. The WHO (World Health Organization) classifies mandibular lesions as benign and malignant, and the updated classification scheme is accessible in the ninth volume of the fourth-edition WHO series [40,41]. Benign tumors represent a broad spectrum of lesions and can be classified as odontogenic, e.g., ameloblastoma, odotoma, and myxoma, and non-odontogenic, e.g., fibro-osseous lesions and giant cell tumors [42]. Benign tumors can grow larger, but do not spread to surrounding tissues, whereas malignant tumors invade, destroy, and spread. The most common malignant tumors include squamous cell carcinomas, osteosarcomas, and giant cell tumors [42]. Tumors represents a major challenge in clinical practice as they lead to facial deformation and functional impairment. The factors influencing the choice of initial tumor treatment are primarily the size (T stage), location (anterior vs. posterior), bone proximity (mandible or maxilla), and histology, including the grade, type, and depth of invasion [43]. Certainly, adequate assessment of the tumor invasion is crucial in diagnosis and optimal surgical treatment planning.

It must be highlighted that, although the mirror image reconstruction technique is an effective tool for implant design, it is not without its drawbacks. A new approach known as cavity-filled reconstruction was, therefore, implemented to address its limitations. The two custom-design reconstruction techniques were compared and evaluated based on their virtual (STL file) and physical (3D-printed) prototypes. The accuracy assessment confirmed that the cavity-filled reconstruction technique provided higher accuracy. In the cavity-filled method, the RMS error was decreased by almost 13-16\% compared to the mirror reconstruction technique. The mean RMS values recorded for mirroring and cavity-filled techniques in the physical prototype analysis were 1.2683 and $1.1019 \mathrm{~mm}$, respectively. Similarly, when the STL files from the mirror and the cavity-filled reconstituted virtual equivalents were reviewed, the RMS error value was reduced from 1.8018 (mirror) to $1.5048 \mathrm{~mm}$ (cavity-filled). The study also established average values of $0.5194 /-0.5392 \mathrm{~mm}$ (AD) for the cavity-filled approach as compared to the mirroring that resulted in the higher values of $0.7441 /-0.8682 \mathrm{~mm}$. Similar trends for AD were observed when STL data were investigated. The AD for the cavity-filled approach was $-0.9675 / 0.6652 \mathrm{~mm}$, and it was quite lower compared to the $-2.9767 / 0.7746 \mathrm{~mm}$ in mirror reconstruction. It was, therefore, noted, based on the results of the 3D and 2D analysis, that the cavity-filled methodology exactly matched the bone contours. It is noteworthy that, in the cavity-filled approach, the bilateral condyle and chin were exactly in the same position unlike in the mirroring technique.

Various techniques are used in the reconstruction of mandibular and maxillary defects, and the most important is mirror reconstruction. Several researchers and surgeons illustrated how the mirror technique can be used successfully in mandibular reconstruction [20,44]. It is also widely used in the reconstruction of custom cranial implants, which replace defective parts of the skull [27,45]. Human skulls are extremely complex and irregular in shape [46]. They are never symmetrically ideal and, thus, the construction of an anatomical frame based on their reference is extremely ambiguous [47]. Throughout clinical practice, the symmetrical plane of the skull is approximated to the mid-sagittal plane and manually calculated using known landmarks [48]. The recognition of the anatomical midpoint used in the mirroring tool is not as simple as one might presume. The slightest deviation of the selected plane would result in discrepancies from the real anatomy. Although this method is simple and uses the patient's actual data as a template, it involves a high degree of user interaction and relies heavily on symmetrical plane computation. On the other hand, the cavity-filled approach is more automated, requiring minimal user intervention. It is faster, easier to use, more straightforward, and more accurate as it requires fewer repetitive tasks. This results in greater control and reliability of the implant performance, thus making it a more efficient reconstruction technique. The current research also demonstrates that the cavity-filled approach would give rise to a more robust implant that would satisfy aesthetic requirements. Contrary to this, the approximation of the midsagittal plane 
using manual landmark points is done in mirror reconstruction, which contributes to deviation and causes implant placement problems. It also reinforces issues in subsequent post-operative recovery and movement of the mandible.

The outcomes of the sample $t$-comparison, as well as one-way ANOVA analysis, of the two reconstruction techniques rejected the null hypothesis of the study. This underlined that the two methods, i.e., mirror and cavity-filled, varied substantially in terms of implant accuracy. It was also noted that the RMS error and AD estimates for the mirror reconstruction technique were greater than for the cavity-filled process. The current study, therefore, compared two reconstruction techniques for accurate mandible restoration, following tumor resection. It also focused on a procedure for estimating the accuracy of the reconstructed mandible models.

\section{Conclusions}

The success of any reconstructive operation depends on the reconstruction technique used in the reenactment of custom-design implants. The mirroring technique is the most commonly used method to recover mandibular defects, but it does have some anomalies and inaccuracies. In this research, a new technique known as the cavity-filled approach was explored as opposed to mirroring reconstruction. To estimate their performance, a 3D comparison was carried out between the reconstructed models of the two modeling techniques, analyzing the deviation. The statistical analysis utilizing hypothesis testing displayed statistically meaningful differences in the two reconstruction techniques. It was discovered on the basis of the 3D deviation study that the new or rather infrequently used cavity-filled technique provided a superior fitting as compared to the mirroring technique. The mirror reconstruction method in 3D-printed models led to a relatively higher RMS error of $1.2683 \mathrm{~mm}$ compared to $1.1019 \mathrm{~mm}$ in the cavity-filled technique due to increased user involvement and often inconsistent symmetrical plane approximation. By using the cavity-filled method, the RMS error decreased by $16.48 \%$ when the STL comparison was implemented. Moreover, the evaluation of 3D-printed models disclosed a notable $13.12 \%$ drop in RMS error with the cavity-filled in contrast to the mirror reconstruction technique. This new design approach demonstrated an integrated framework for the reconstruction of personalized mandibular implants, which provides greater facial symmetry and reduces operating time by minimizing revisions. However, the cavity-filled technique is a great and promising technique which might be limited to expanding lesions that have intact or minimal cortical wall destruction. Conversely, in the case of severe destructive lesions with distorted anatomy and loss of cortical plates or in the case of pathological mandibular fractures with loss of pre-tumor dimensions, applying such a technique might be challenging.

Author Contributions: K.M., conceptualization and experiments; K.M., methodology and draft preparation; S.H.M., analysis and investigation; K.M. and S.H.M., data curation, resources, and investigation; M.A., project administration and funding acquisition; S.R., validation; W.A. and O.A., visualization and supervision; K.M., writing; M.A. and S.R., review and editing. All authors read and agreed to the published version of the manuscript.

Funding: This research was funded by the Deanship of Scientific Research at King Saud University through Research Group no. RG-1440-075.

Acknowledgments: The authors extend their appreciation to the Deanship of Scientific Research at King Saud University for funding this work through Research Group no. RG-1440-075.

Conflicts of Interest: The authors declare no conflict of interest.

Ethical Statement: Ethical committee at the College of Dentistry Research Center, King Saud University, Riyadh, Saudi Arabia (Registration \# FR 0467).

\section{References}

1. Torroni, A.; Marianetti, T.; Romandini, M.; Gasparini, G.; Cervelli, D.; Pelo, S. Mandibular Reconstruction with Different Techniques. J. Craniofacial Surg. 2015, 26, 885-890. [CrossRef] [PubMed]

2. Kumar, B.P.; Venkatesh, V.; Kumar, K.A.J.; Yadav, B.Y;; Mohan, S.R. Mandibular Reconstruction: Overview. J. Maxillofac. Oral Surg. 2016, 15, 425-441. [CrossRef] [PubMed] 
3. Zeller, A.; Neuhaus, M.T.; Weissbach, L.V.M.; Rana, M.; Dhawan, A.; Eckstein, F.M.; Gellrich, N.C.; Zimmerer, R.M. Patient-Specific Mandibular Reconstruction Plates Increase Accuracy and Long-Term Stability in Immediate Alloplastic Reconstruction of Segmental Mandibular Defects. J. Maxillofac. Oral Surg. 2020. [CrossRef]

4. Parthasarathy, J. 3D modeling, custom implants and its future perspectives in craniofacial surgery. Ann. Maxillofac. Surg. 2013, 4, 9-18. [CrossRef]

5. Bowers, C.A.; McMullin, J.H.; Brimley, C.; Etherington, L.; Siddiqi, F.A.; Riva-Cambrin, J. Minimizing bone gaps when using custom pediatric cranial implants is associated with implant success. J. Neurosurg. Pediatrics 2015, 16, 439-444. [CrossRef]

6. Cassoni, A.; Brauner, E.; Pucci, R.; Terenzi, V.; Mangini, N.; Battisti, A.; Monaca, M.D.; Ciolfi, A.; Laudoni, F.; Di Carlo, S.; et al. Head and Neck Osteosarcoma-The Ongoing Challenge about Reconstruction and Dental Rehabilitation. Cancers 2020, 12, 1948. [CrossRef]

7. Meyer-Marcotty, P.; Alpers, G.W.; Gerdes, A.B.M.; Stellzig-Eisenhauer, A. Impact of facial asymmetry in visual perception: A 3-dimensional data analysis. Am. J. Orthod. Dentofac. Orthop. 2010, 137, 168.e1-168.e8. [CrossRef]

8. Metzler, P.; Geiger, E.J.; Alcon, A.; Ma, X.; Steinbacher, D.M. Three-dimensional virtual surgery accuracy for free fibula mandibular reconstruction: Planned versus actual results. J. Oral Maxillofac. Surg. 2014, 72, 2601-2612. [CrossRef]

9. Alasseri, N.; Alasraj, A. Patient-specific implants for maxillofacial defects: Challenges and solutions. Maxillofac. Plast. Reconstr. Surg. 2020, 42, 15. [CrossRef]

10. Huotilainen, E.; Jaanimets, R.; Valášek, J.; Marcián, P.; Salmi, M.; Tuomi, J.; Mäkitie, A.; Wolff, J. Inaccuracies in additive manufactured medical skull models caused by the DICOM to STL conversion process. J. Craniomaxillofac. Surg. 2014, 42, e259-e265. [CrossRef]

11. Davim, J.P. Additive and Subtractive Manufacturing: Emergent Technologies; Walter de Gruyter GmbH \& Co KG: Berlin, Germany, 2020.

12. Boyette, J.R.; Pemberton, J.D.; Bonilla-Velez, J. Management of orbital fractures: Challenges and solutions. Clin. Ophthalmol. 2015, 9, 2127-2137. [CrossRef] [PubMed]

13. Goormans, F.; Sun, Y.; Bila, M.; Schoenaers, J.; Geusens, J.; Lübbers, h.-t.; Coucke, W.; Politis, C. Accuracy of computer-assisted mandibular reconstructions with free fibula flap: Results of a single-center series. Oral Oncol. 2019, 97, 69-75. [CrossRef] [PubMed]

14. Lancellotta, V.; Pagano, S.; Tagliaferri, L.; Piergentini, M.; Ricci, A.; Montecchiani, S.; Saldi, S.; Chierchini, S.; Chierchini, S.; Cianetti, S.; et al. Individual 3-dimensional printed mold for treating hard palate carcinoma with brachytherapy: A clinical report. J. Prosthet. Dent. 2019, 121, 690-693. [CrossRef] [PubMed]

15. Pagano, S.; Moretti, M.; Marsili, R.; Ricci, A.; Barraco, G.; Cianetti, S. Evaluation of the Accuracy of Four Digital Methods by Linear and Volumetric Analysis of Dental Impressions. Materials 2019, 12, 1958. [CrossRef]

16. Kirke, D.N.; Owen, R.P.; Carrao, V.; Miles, B.A.; Kass, J.I. Using 3D computer planning for complex reconstruction of mandibular defects. Cancers Head Neck 2016, 1, 17. [CrossRef]

17. Bartier, S.; Mazzaschi, O.; Benichou, L.; Sauvaget, E. Computer-assisted versus traditional technique in fibular free-flap mandibular reconstruction: A CT symmetry study. Eur. Ann. Otorhinolaryngol. Head Neck Dis. 2020. [CrossRef]

18. Mascha, F.; Winter, K.; Pietzka, S.; Heufelder, M.; Schramm, A.; Wilde, F. Accuracy of computer-assisted mandibular reconstructions using patient-specific implants in combination with CAD/CAM fabricated transfer keys. J. Cranio-Maxillofac. Surg. 2017, 45, 1884-1897. [CrossRef]

19. Yang, W.-F.; Zang, C.-Y.; Choi, W.S.; Zhu, W.-Y.; Li, D.T.S.; Chen, X.-S.; Du, R.; Su, Y.-X. A novel 'surgeon-dominated' approach to the design of 3D-printed patient-specific surgical plates in mandibular reconstruction: A proof-of-concept study. Int. J. Oral Maxillofac. Surg. 2020, 49, 13-21. [CrossRef]

20. Davies, J.C.; Chan, H.H.L.; Jozaghi, Y.; Goldstein, D.P.; Irish, J.C. Analysis of simulated mandibular reconstruction using a segmental mirroring technique. J. Craniomaxillofac. Surg. 2019, 47, 468-472. [CrossRef]

21. Mahendru, S.; Jain, R.; Aggarwal, A.; Aulakh, H.S.; Jain, A.; Khazanchi, R.K.; Sarin, D. CAD-CAM vs. conventional technique for mandibular reconstruction with free fibula flap: A comparison of outcomes. Surg. Oncol. 2020, 34, 284-291. [CrossRef] 
22. Darwich, K.; Ismail, M.B.; Al-Mozaiek, M.Y.A.-S.; Alhelwani, A. Reconstruction of mandible using a computer-designed 3D-printed patient-specific titanium implant: A case report. Oral Maxillofac. Surg. 2020. [CrossRef] [PubMed]

23. Linares, O.C.; Bianchi, J.; Raveli, D.; Neto, J.B.; Hamann, B. Mandible and skull segmentation in cone beam computed tomography using super-voxels and graph clustering. Vis. Comput. 2019, 35, 1461-1474. [CrossRef]

24. Qiu, B.; Guo, J.; Kraeima, J.; Glas, H.H.; Borra, R.J.H.; Witjes, M.J.H.; van Ooijen, P.M.A. Automatic segmentation of the mandible from computed tomography scans for 3D virtual surgical planning using the convolutional neural network. Phys. Med. Biol. 2019, 64, 175020. [CrossRef] [PubMed]

25. Abbasi, A.J.; Parvin, M.; Bashiri, S. Innovative use of a stereolithographic model together with the mirror image technique to reconstruct a defect in mandibular continuity. Br. J. Oral Maxillofac. Surg. 2018, 56, 887-889. [CrossRef]

26. Lin, H.; Zhu, P.; Lin, Q.; Huang, X.; Xu, Y.; Yang, X. Comprehensive Analysis of Mandibular Residual Asymmetry after Bilateral Sagittal Split Ramus Osteotomy Correction of Menton Point Deviation. PLoS ONE 2016, 11, e0161601. [CrossRef]

27. Moiduddin, K.; Darwish, S.; Al-Ahmari, A.; ElWatidy, S.; Mohammad, A.; Ameen, W. Structural and mechanical characterization of custom design cranial implant created using additive manufacturing. Electron. J. Biotechnol. 2017, 29, 22-31. [CrossRef]

28. Hatamleh, M.M.; Bhamrah, G.; Ryba, F.; Mack, G.; Huppa, C. Simultaneous Computer-Aided Design/Computer-Aided Manufacture Bimaxillary Orthognathic Surgery and Mandibular Reconstruction Using Selective-Laser Sintered Titanium Implant. J. Craniofacial Surg. 2016, 27, 1810-1814. [CrossRef]

29. Scolozzi, P. Maxillofacial reconstruction using polyetheretherketone patient-specific implants by 'mirroring' computational planning. Aesthetic Plast. Surg. 2012, 36, 660-665. [CrossRef]

30. Neyman, J.; Pearson, E.S.; Pearson, K. On the problem of the most efficient tests of statistical hypotheses. Philos. Trans. Royal Soc. Lond. Ser. A 1933, 231, 289-337. [CrossRef]

31. Giudice, A.L.; Ronsivalle, V.; Grippaudo, C.; Lucchese, A.; Muraglie, S.; Lagraere, M.O.; Isola, G. One Step before 3D Printing-Evaluation of Imaging Software Accuracy for 3-Dimensional Analysis of the Mandible: A Comparative Study Using a Surface-to-Surface Matching Technique. Materials 2020, 13, 2798. [CrossRef]

32. Mian, S.H.; Mannan, M.A.; Al-Ahmari, A.M. The influence of surface topology on the quality of the point cloud data acquired with laser line scanning probe. Sens. Rev. 2014, 34, 255-265. [CrossRef]

33. Hague, R.J.M.; Reeves, P.E. Rapid Prototyping, Tooling and Manufacturing; iSmithers Rapra Publishing: Shropshire, UK, 2000.

34. Material Properties of ABS-Acrylonitrile-Butadlene-Styrene. Dielectric Manufacturing. Available online: https://dielectricmfg.com/knowledge-base/abs/ (accessed on 31 January 2020).

35. Oropallo, W.; Piegl, L.A. Ten challenges in 3D printing. Eng. Comput. 2016, 32, 135-148. [CrossRef]

36. Umer, U.; Ameen, W.; Abidi, M.H.; Moiduddin, K.; Alkhalefah, H.; Alkahtani, M.; Al-Ahmari, A. Modeling the Effect of Different Support Structures in Electron Beam Melting of Titanium Alloy Using Finite Element Models. Metals 2019, 9, 806. [CrossRef]

37. About Arcam. GE Additive. Available online: https://www.ge.com/additive/who-we-are/about-arcam (accessed on 4 March 2020).

38. Calignano, F. Design optimization of supports for overhanging structures in aluminum and titanium alloys by selective laser melting. Mater. Des. 2014, 64, 203-213. [CrossRef]

39. Martinez-Marquez, D.; Mirnajafizadeh, A.; Carty, C.P.; Stewart, R.A. Application of quality by design for 3D printed bone prostheses and scaffolds. PLOS ONE 2018, 13. [CrossRef]

40. Adel, E.-N.K.; John, C.K.C.; Jennifer, G.; Takashi, T.; Pieter, J.S. WHO Classification of Head and Neck Tumours, 4th ed.; International Agency for Research on Cancer (IACR): Lyon, France, 2017; Volume 9.

41. Tekkeşin, M.S.; Wright, J. The World Health Organization Classification of Odontogenic Lesions: A Summary of the Changes of the 2017 (4th) Edition. Turk. J. Pathol. 2013, 34. [CrossRef]

42. Dunfee, B.L.; Sakai, O.; Pistey, R.; Gohel, A. Radiologic and Pathologic Characteristics of Benign and Malignant Lesions of the Mandible. RadioGraphics 2006, 26, 1751-1768. [CrossRef]

43. Shah, J. Cancer of the Head and Neck, Mandible Reconstruction. Cancer Head Neck 2013, 19, 355-375. 
44. Essig, H.; Rana, M.; Kokemueller, H.; von See, C.; Ruecker, M.; Tavassol, F.; Gellrich, N.-C. Pre-operative planning for mandibular reconstruction-A full digital planning workflow resulting in a patient specific reconstruction. Head Neck Oncol. 2011, 3, 45. [CrossRef]

45. Jardini, A.L.; Larosa, M.A.; Filho, R.M.; de Carvalho Zavaglia, C.A.; Bernardes, L.F.; Lambert, C.S.; Calderoni, D.R.; Kharmandayan, P. Cranial reconstruction: 3D biomodel and custom-built implant created using additive manufacturing. J. Cranio-Maxillofac. Surg. 2014, 42, 1877-1884. [CrossRef]

46. White, T.D.; Folkens, P.A. Chapter 7-SKULL. In The Human Bone Manual; White, T.D., Folkens, P.A., Eds.; Academic Press: San Diego, CA, USA, 2005; pp. 75-126.

47. Ferrario, V.F.; Sforza, C.; Ciusa, V.; Dellavia, C.; Tartaglia, G.M. The effect of sex and age on facial asymmetry in healthy subjects: A cross-sectional study from adolescence to mid-adulthood. J. Oral Maxillofac. Surg. 2001, 59, 382-388. [CrossRef] [PubMed]

48. Yáñez-Vico, R.M.; Iglesias-Linares, A.; Torres-Lagares, D.; Gutiérrez-Pérez, J.L.; Solano-Reina, E. Three-dimensional evaluation of craniofacial asymmetry: An analysis using computed tomography. Clin. Oral Investig. 2011, 15, 729-736. [CrossRef] [PubMed]

C 2020 by the authors. Licensee MDPI, Basel, Switzerland. This article is an open access article distributed under the terms and conditions of the Creative Commons Attribution (CC BY) license (http://creativecommons.org/licenses/by/4.0/). 